\title{
Analysis of Magnetic Permeability Spectra of Metamaterials Composed of Cut Wire Pairs by Circuit Theory
}

\author{
Jun-Hee Lim and Sung-Soo Kim* \\ Department of Advanced Materials Engineering, Chungbuk National University, Cheongju 28644, Korea
}

(Received 26 March 2016, Received in final form 26 April 2016, Accepted 26 April 2016)

\begin{abstract}
Retrieving the equivalent electromagnetic parameters (permittivity and permeability) plays an important role in the research and application of metamaterials. Frequency dispersion of magnetic permeability has been theoretically predicted in a metamaterial composed of cut wire pairs (CWP) separated by dielectric substrate on the basis of circuit theory. Magnetic resonance resulting from antiparallel currents between the CWP is observed at the frequency of minimum reflection loss (corresponding to absorption peak) and effective resonator size can be determined. Having calculated the circuit parameters (inductance $L$, capacitance $C$ ) and resonance frequency from CWP dimension, the frequency dispersion of permeability of Lorentz like magnetic response can be predicted. The simulated resonance frequency and permeability spectra can be explained well on the basis of the circuit theory of an $R L C$ resonator.
\end{abstract}

Keywords : permeability, metamaterials, circuit theory, magnetic resonance

\section{Introduction}

Electromagnetic metamaterials are arrays of structured subwavelength elements which may be described as effective materials via $\varepsilon(\omega)$ and $\mu(\omega)$, the electric permittivity and magnetic permeability, respectively. Initial interest in metamaterials arose due to their ability to exhibit exotic electromagnetic effects (such as negative index of refraction), which can be achieved with artificial materials having simultaneously negative electric permittivity and magnetic permeability [1-4]. Although the first experimental metamaterials demonstrated by Smith et al. [3] have a three-dimensional array of thin wires and split ring resonators, most of the metamaterials utilize the planar three-layers structure (two metallic layers separated by a dielectric substrate) [5-7]. The top and bottom layer consists of an electric resonator (ER) which provides the electric response $(\varepsilon)$ by coupling strongly to incident electric field at a certain resonance frequency. Magnetic coupling is achieved via antiparallel currents in the ER conductor on top layer and ground conductor on bottom layer. An incident time-varying magnetic field may couple to these antiparallel currents, thus yielding a Lorentz like

(C)The Korean Magnetics Society. All rights reserved.

*Corresponding author: Tel: +82-43-261-2418

Fax:+82-43-271-3222, e-mail: sskim@cbnu.ac.kr magnetic response $(\mu)$.

Accurately retrieving the equivalent electromagnetic parameters (permittivity and permeability) plays an important role in the research and application of metamaterials. One of the commonly adopted approaches to determine such parameters is to retrieve them from the simulated or measured transmission and reflection coefficients of a metamaterial slab, which is the so-called the S-parameter retrieval [8-10]. The behavior of metamaterials can be sometimes modeled by equivalent electrical circuits. The response of an artificial magnetic inclusion is due to resonant oscillating currents, and a common way to describe this is to model the inclusion by an equivalent effective resistor-inductor-capacitor $(R L C)$ circuit [11, 12]. Using this theory, one can obtain an expression for the magnetic moment and the frequency dependence of the effective magnetic permeability $\mu(\omega)$.

In this study, frequency dispersion of magnetic permeability was investigated in a metamaterial composed of cut wire pairs (CWP) separated by dielectric substrate on the basis of circuit theory. The reason for choosing the CWP for this study is that they are simple and easy to design and comprehensively studied in many previous studies [12-14]. Magnetic resonance resulting from antiparallel currents between the CWP was predicted by computational tools. The simulated resonance frequency and permeability spectra can be explained well on the 
basis of the circuit theory of an $R L C$ resonator.

\section{Circuit Theory}

A system of cut wire pairs with width $w$ and length $l$ separated by dielectric substrate (with thickness $t_{b}$ ) is illustrated in Fig. 1(a). It is excited by a magnetic field which has the harmonic time dependence $H=H_{0} e^{j \omega t}$ and applied perpendicularly to the pair plane. At the magnetic resonance of the structure, resonant antiparallel currents are excited in the two wires of the pair, as shown in Fig. 1(b), creating an effective loop current. Moreover, this current results in accumulation of opposite charges at the two upper (and at the two lower) sides of the pair, creating capacitance there. The structure thus can be approximated by an equivalent circuit (as is shown in Fig. 1(c)), of inductance $L$, capacitance $C=C_{1} / 2$, and resistance $R$.

Under these conditions, the sum of the voltage drop over the inductance, capacitance, and resistance, equal the voltage induced by the external magnetic flux,

$$
L \frac{d I}{d t}+\frac{1}{C} \int I d t+I R=U_{\text {ind }}=-\frac{d \varphi}{d t}
$$

where $\varphi$ is the external magnetic flux $\varphi=\mu_{0} l t_{b} H$, and $I$ is the current. Having the solution for the current, one can easily obtain the magnetic permeability $\mu$ as a function of angular frequency $\omega$ [15], through obtaining the magnetization results,

$$
\mu(\omega)=\mu_{0}\left[1-\frac{\left(\frac{1}{V_{u c}}\right)\left(\frac{\mu_{0}\left(l t_{b}\right)^{2}}{L}\right) \omega^{2}}{\omega^{2}-\omega_{L C}^{2}-j \omega \gamma}\right]
$$

where $\mu_{0}=$ permeability of vacuum, $V_{u c}=$ unit cell volume,

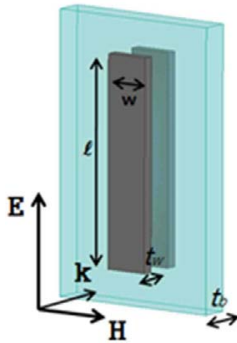

(a)

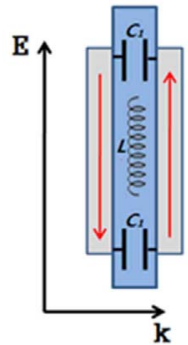

(b)

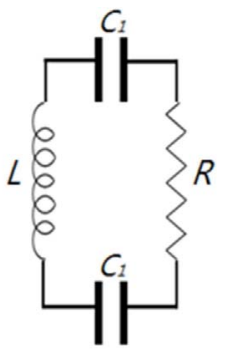

(c)
Fig. 1. (Color online) Schematic description of (a) a typical artificial magnetic structure formed by a pair of cut wires and excited by an external electromagnetic field, (b) E-k cross-section of the structure shown in (a), and (c) its equivalent $R L C$ circuit.
$\omega_{L C}=1 / \sqrt{L C}=$ resonance frequency and $\gamma=R / L=$ damping factor. For a solenoid of area $l t_{b}$ and length $w$, the magnetic field inductance $L$ is given by $L=\mu_{0} l t_{b} / w$, and the $\mu$ expression turns to

$$
\begin{aligned}
& \frac{\mu(\omega)}{\mu_{0}}=1-\frac{F \omega^{2}}{\omega^{2}-\omega_{L C}^{2}-j \omega \gamma} \\
& F=\frac{l t_{b} w}{V_{u c}}=\text { (inter-pair volume)/(unit cell volume) }
\end{aligned}
$$

This formula represents a Lorenz oscillator resonance for a magnetic atom. Frequency dispersion of magnetic permeability depends on CWP filling factor $(F)$, resonance frequency $\left(\omega_{L C}\right)$, and damping factor $(=R / L)$.

\section{Simulation Model}

Figure 2 shows the structure of a metamaterial composed of CWP separated by dielectric substrate. An electromagnetic wave (with wave vector $\mathrm{k}$ ) is incident normal to the composite structure. The electric field (E) is polarized parallel to the long axis of the SCW, and the magnetic field $(\mathrm{H})$ is parallel to the plane of the substrate, as shown in Fig. 2(a). The dimensions of the unit cell and CWP are given in Fig. 2(b). The substrate material is FR4 with relative dielectric permittivity $\varepsilon_{r}=4.1+\mathrm{j} 0.03$. The metal on the top and bottom layer is copper with electrical conductivity $\sigma=5.8 \times 10^{7} \mathrm{~S} / \mathrm{m}$ and thickness $t_{m}=$ $0.035 \mathrm{~mm}$.

Computational tools (HFSS 13.0) were used to model the interaction between electromagnetic waves and the materials in the structures shown in Fig. 2. The simulation output included the complex scattering parameters, the transmission coefficient $\left(\mathrm{S}_{21}\right)$ and reflection coefficient $\left(\mathrm{S}_{11}\right)$. Another parameter of interest was the surface (a)

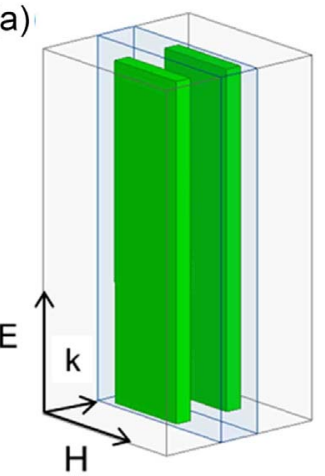

(b)

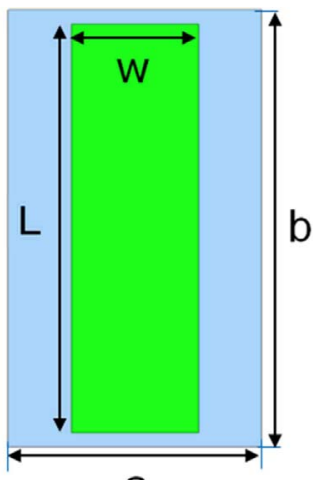

a
Fig. 2. (Color online) Schematic description of (a) cut wire pair separated by dielectric substrate and (b) their dimension $(\mathrm{a}=16 \mathrm{~mm}, \mathrm{~b}=32 \mathrm{~mm}, \mathrm{w}=8 \mathrm{~mm}, \mathrm{~L}=30 \mathrm{~mm})$. 
currents, which may be used to show the resonating behavior of the metallic portions of the CWP.

\section{Results and Discussion}

Figure 3 shows the simulation result for reflection loss, transmission loss, and power absorption for CWP with a unit cell $(\mathrm{a}=16 \mathrm{~mm}, \mathrm{~b}=32 \mathrm{~mm})$ and $\mathrm{CWP}(\mathrm{w}=8 \mathrm{~mm}$, $\mathrm{L}=30 \mathrm{~mm}$ ). The substrate thickness is $t_{b}=0.53 \mathrm{~mm}$. Reflection loss $\left(\mathrm{S}_{11}\right)$ shows the resonance peak at 7.52
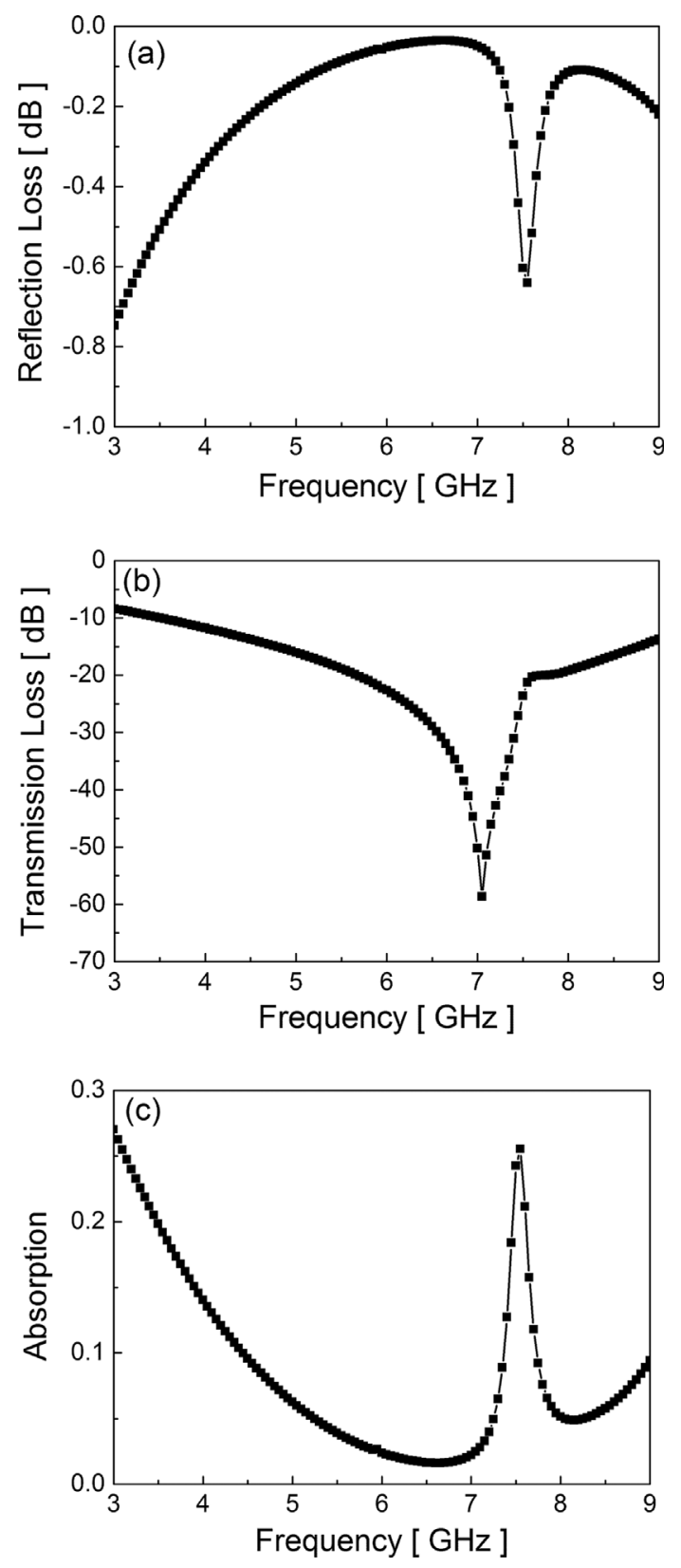

Fig. 3. Simulation results for the CWP metamaterial absorber: (a) reflection loss, (b) transmission loss, and (c) power absorption.
$\mathrm{GHz}$, as shown in Fig. 3(a). Transmission loss $\left(\mathrm{S}_{21}\right)$ has a low value, less than $-10 \mathrm{~dB}$ for all frequencies, and shows a resonance peak at $7.08 \mathrm{GHz}$ (Fig. 3(b)). Power absorption (represented by $A=1-\left|S_{11}\right|^{2}-\left|S_{21}\right|^{2}$ ) shows a peak value of $25 \%$ at $7.52 \mathrm{GHz}$ corresponding to the reflection loss minimum.

Figure 4 shows the current density distribution at the resonance frequencies $(7.08 \mathrm{GHz}$ and $7.52 \mathrm{GHz})$. At 7.08 $\mathrm{GHz}$, parallel currents are observed at CWP as shown in Fig. 4(a), indicating the electrical resonance of symmetric plasmon mode [6]. On the while, antiparallel currents are clearly shown in the CWP at $7.52 \mathrm{GHz}$, as depicted in Fig. 4(b). An incident time-varying magnetic field may couple to these antiparallel currents, thus yielding a Lorentz-like magnetic response [6, 11]. The magnetic resonance occurs at three parts in one wire strip, and, therefore, the effective resonator length is $l=\mathrm{L} / 3$.

The magnetic resonance frequency $\left(f_{m}\right)$ is given by Eq. (5), and is inversely proportional to the inductance $(L)$ and capacitance $(C)$ of the CWP, which are given by Eqs.

(a)
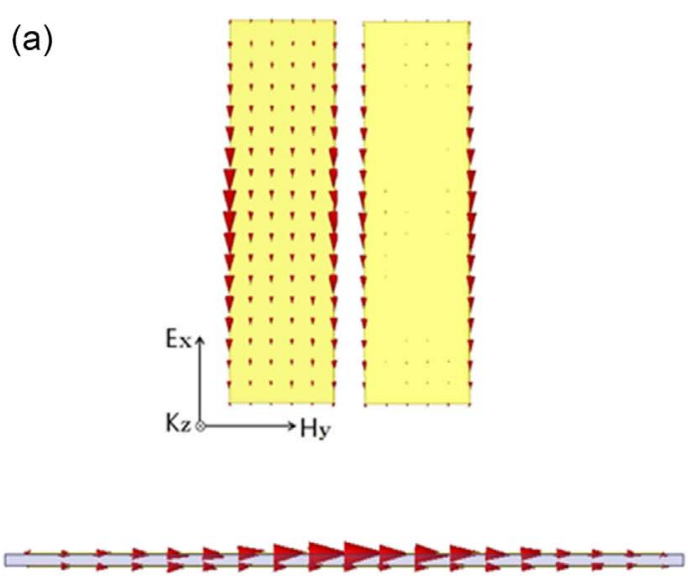

(b)
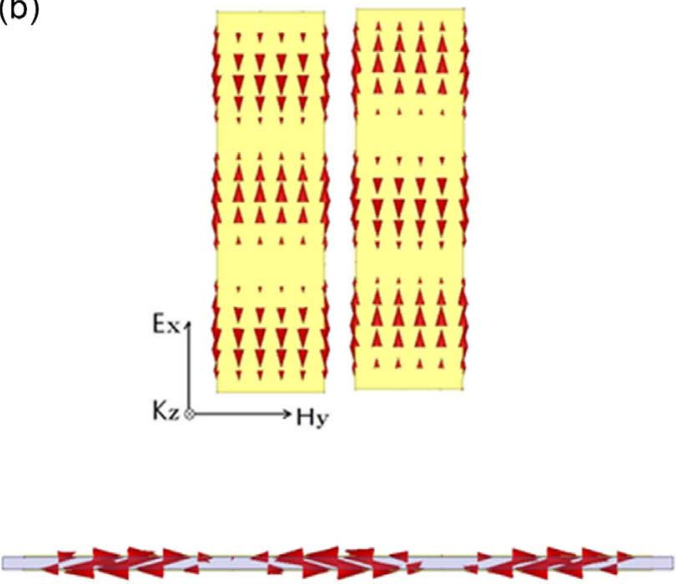

Fig. 4. (Color online) Plane and side view of current density distribution in cut wire pair at the resonance frequencies: (a) $7.08 \mathrm{GHz}$, (b) $7.52 \mathrm{GHz}$. 
(6) and (7).

$$
\begin{aligned}
& f_{m}=1 / 2 \pi \sqrt{L C} \\
& L \cong \mu\left(\frac{t_{b}}{w}\right) l \\
& C \cong \varepsilon w(0.2 l) / t_{b}
\end{aligned}
$$

where $\mu$ : permeability, $\varepsilon$ : permittivity, $t_{b}$ : substrate thickness, $w$ : resonator width, $l$ : resonator length. Since the electric field is confined mainly to the edge of the resonator, the effective length for capacitance between CWP is reduced to $0.2 l$ [12]. For the magnetic resonator with dimensions $\left(l=\mathrm{L} / 3=10 \mathrm{~mm}, w=8 \mathrm{~mm}, t_{b}=0.53\right.$ $\mathrm{mm})$ and material parameters of FR4 substrate $\left(\varepsilon_{r}=4.1+\right.$ $\mathrm{j} 0.03$ ), the inductance and capacitance are calculated to be $L=0.832 \times 10^{-9}[\mathrm{H}], C=0.548 \times 10^{-12}[\mathrm{~F}]$, respectively. Using these values, the magnetic resonance frequency is calculated to be $f_{m}=7.47 \mathrm{GHz}$, which is consistent with the simulation result for absorption frequency.

Having calculated the resonance frequency $\left(f_{m}=7.47\right.$ $\mathrm{GHz}), \mathrm{CWP}$ filling factor $\left(F=l t_{b} w / V_{u c}=0.156\right)$, inductance $\left(L=0.832 \times 10^{-9}[\mathrm{H}]\right)$, and resistance $\left(R=10^{-5} \sim 10^{0} \Omega\right)$,
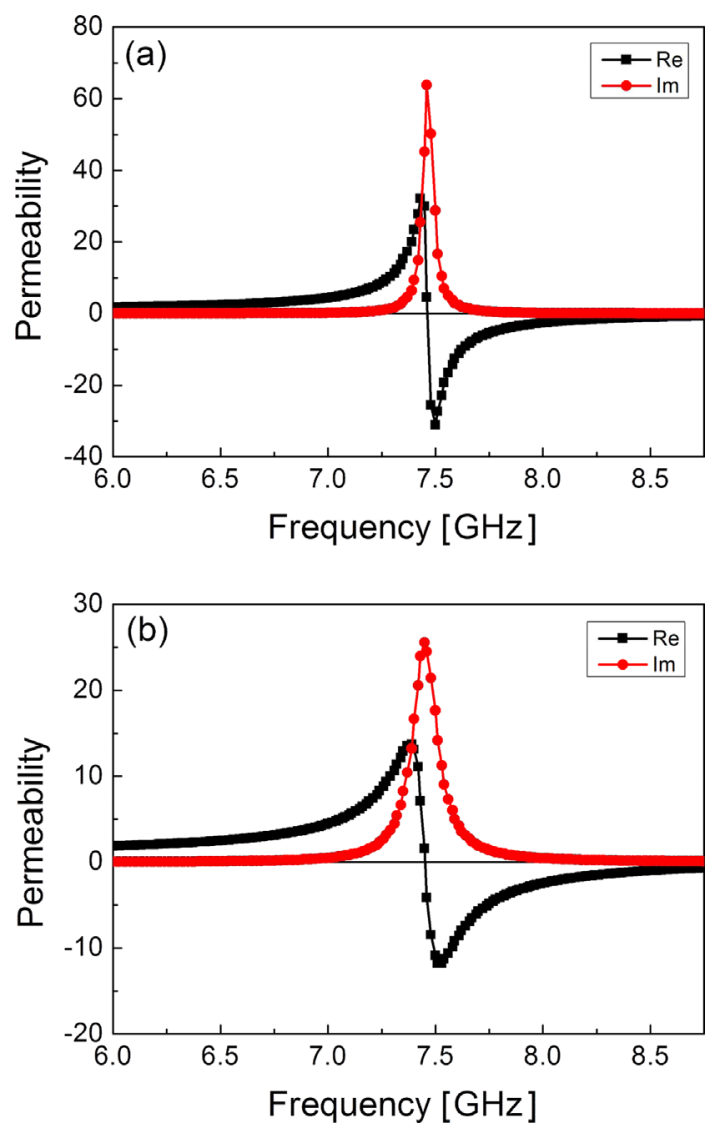

Fig. 5. (Color online) Real and imaginary part of complex permeability of cut wire pair with dimension shown in Fig. 2 with variation of surface resistance: (a) $R=10^{-5} \Omega$, (b) $R=10^{0} \Omega$. frequency dispersion of permeability can be predicted using Eq. (3). Figure 5 shows the complex permeability of CWP with increasing surface resistance of conductor. A typical pattern of Lorentz like magnetic response is depicted: real part of permeability $(\operatorname{Re}(\mu))$ transits from positive to negative values through the resonance frequency and imaginary part $(\operatorname{Im}(\mu))$ shows a peak there. For a small value of surface resistance $R=10^{-5} \Omega$, a sharp and narrow magnetic resonance is predicted, as shown in Fig. 5(a). If the surface resistance increases to $10^{\circ} \Omega$, the resonance is more dispersive and resonance peak is reduced, as shown in Fig. 5(b). The resonance frequency is invariant with surface resistance and the metamaterial regime with negative value of $\operatorname{Re}(\mu)$ is found in the frequency region above $f_{m}$.

\section{Conclusion}

Frequency dispersion of magnetic permeability was theoretically predicted in a metamaterial composed of cut wire pairs (CWP) separated by dielectric substrate on the basis of circuit theory. Magnetic resonance resulting from antiparallel currents between the CWP was observed at the frequency of minimum reflection loss (corresponding to absorption peak) and effective resonator size could be determined. Having calculated the circuit parameters (inductance $L$, capacitance $C$ ) and resonance frequency from CWP dimension, the frequency dispersion of permeability of Lorentz like magnetic response could be predicted. The simulated resonance frequency and permeability spectra can be explained well on the basis of the circuit theory of an $R L C$ resonator. The present analysis technique based on the circuit theory can be applied to other metamaterial structures.

\section{Acknowledgments}

This research was supported by the Basic Research Program through the National Research Foundation of Korea (grant number: 2013R1A1A2A10005073).

\section{References}

[1] J. B. Pendry, A. J. Holden, W. J. Stewart, and I. Youngs, Phys. Rev. Lett. 76, 4773 (1996).

[2] J. B. Pendry, A. J. Holden, D. J. Robbins, and W. J. Stewart, IEEE Trans. Microwave Theory and Techniques, 47, 2075 (1999).

[3] D. R. Smith, W. J. Padilla, D. C. Vier, S. C. NematNasser, and S. Schultz, Phys. Rev. Lett. 84, 4184 (2000).

[4] R. A. Shelby, D. R. Smith, and S. Schultz, Science 292, 77 (2001). 
[5] V. D. Lam, J. B. Kim, S. J. Lee, and Y. P. Lee, Opt. Express 15, 16651 (2007).

[6] N. T. Tung, V. T. T. Thuy, J. W. Park, J. Y. Rhee, and Y. Lee, J. Appl. Phys. 107, 023530 (2010).

[7] N. I. Landy, S. Sajuyigbe, J. J. Mock, D. R. Smith, and W. J. Padilla, Phys. Rev. Lett. 100, 207402 (2008).

[8] D. R. Smith, S. Schultz, P. Markoš, and C. M. Soukoulis, Phys. Rev. B 65, 195104 (2002).

[9] D. R. Smith, D. C. Vier, N. Kroll, and S. Schultz, Appl. Phys. Lett. 77, 2246 (2000).

[10] D. R. Smith, D. C. Vier, T. Koschny, and C. M. Soukoulis, Phys. Rev. E 71, 036617 (2005).
[11] A. Ourir and H. H. Ouslimani, Appl. Phys. Lett. 98, 113505 (2011).

[12] J. Zhou, E. N. Economon, T. Koschny, and C. M. Soukoulis, Opt. Lett. 31, 3620 (2006).

[13] B. Kanté, S. N. Burokur, A. Sellier, A. de Lustrac, and J.M. Lourtioz, Phys. Rev. B 79, 075121 (2009).

[14] A. Ourir1 and H. H. Ouslimani, Appl. Phys. Lett. 98, 113505 (2011).

[15] Nanostructured Metamaterials - Exchange between experts in electromagnetics and material science, edited by A. F. de Baas, Luxembourg: Publications Office of the European Union (2010) pp. 34-36. 